\title{
Designing a biomedical coding program focused on inclusivity and agency
}

To the Editor - Last year, scientists across the world participated in the 10 June protests and used hashtags such as \#ShutDownAcademia, \#ShutDownSTEM and \#Strike4BlackLife, which called for non-Black academics to take action towards eradicating anti-Black racism in academia, including the disciplines of science, technology, engineering and math (STEM). This resulted in an outpouring of stories by Black academics detailing the racist experiences that they have endured. Their stories underlie an urgent need for transforming academic structures to be more equitable.

To make a positive change towards this goal, last year we created the Virtual BUILD Research Collaboratory (VBRC), an online summer program focused on diversity and inclusion. Summer programs are crucial for students as they are a critical step towards building scientific careers. The VBRC was developed out of the SF BUILD (Building Infrastructure Leading to Diversity), a six-year-old program for undergraduate students from diverse backgrounds in biomedical research, typically held in person. Given the disruptions caused by the pandemic, our team had to transform the regular in-person offering into an eight-week online summer coding program, which took place from 22 June-13 August 2020. As we were able to pivot rapidly to a new solution, we offered it to nine sister National Institutes of Health (NIH) BUILD sites across the country. The objective of the virtual eight-week summer coding program was to teach students to use the statistical programming language and software R/RStudio to perform research related to health disparities due to COVID-19.

Purposeful racial representation and comprehensive inclusivity were at the center of the VBRC. The program had a total of 65 students, who were on average 23.7 years old (standard deviation of 5.25), 73\% cisgender female, 37\% Black, 39\% Latinx, 14\% non-Black/Latinx, and 10\% Asian or other. Most of the students had no prior experience with coding, and many were majoring in biomedical sciences at minority-serving institutions across the United States.

Additionally, students participated in scholarly engagement discussions with 15 scientists researching COVID-19 or critical race theory, most of whom were from historically underrepresented communities. When engaging with students, the faculty not only explained their current work in response to COVID-19, but also described their scientific journey. This scholarly engagement fostered a collaborative learning environment that empowered students to consider their experiences and culture as valuable sources of knowledge to advance scientific inquiry.

An intentional non-hierarchical teaching environment focused on the students' interests was established. The VBRC program combined large group sessions (with all 65 students) with smaller breakout groups (5-7 students) led by near peer mentors (NPMs), all of them occurring online. The relationships that NPMs built with students were vital to the success of our program. NPMs were all graduate students and Black, Indigenous and people of color (BIPOC) (67\% Latinx, 11\% Black, $11 \%$ Asian American, and 11\% Indian American), to which most of the students of the program could relate. NPMs also had learned to code within the last 2-4 years: they could easily relate to students facing challenges while learning to code and could use this experience to better guide students in this process. NPMs allowed us to stay away from the pitfalls of traditional teacher-student power dynamics. We felt that this model was important in creating an environment where students felt comfortable in asking questions in real-time, allowing them to get to a place of understanding more quickly.

VBRC emphasized real learning, motivation and presence, not grades. All of the students were living the reality of how COVID-19 was affecting communities of color $^{1}$, so picking this timely, relevant and relatable theme was very important. The focus was on engaging with real problems in real-time, where students used publicly available data to understand how health disparities were affecting their communities. The final deliverable of the course was a presentation on COVID-19 and race/ ethnicity: 54 team presentations were delivered with a great deal of enthusiasm and included a message to communities of color about the results on a myriad of topics (wealth disparities, prisons, Immigration and Customs Enforcement detention facilities, race/ethnicity, mental health and their relationship to COVID-19). For example, one message included the importance of COVID-19 testing for communities of color along with the availability of testing sites in the local communities.

Lastly, VBRC faculty practiced cultural humility, with an openness to learning from students about which teaching methods did not work and adapting to students' learning styles. Adaptations varied depending on what the students, not faculty or NPMs, felt worked best. An example of this was the original curriculum, which used open-source didactic lectures to teach the $\mathrm{R}$ programming language. Students provided feedback that the course did not sufficiently teach the basics needed to be confident using R, and the open-source lectures were eliminated. Perhaps in a less inclusive format, students would be expected to adapt to the preset curriculum even if it did not meet the students' needs. In VBRC, we listened to what was working and what was not working and adapted. The VBRC coordinators and NPMs then collaborated to make worksheets that introduced the basics (variables, scripts, comments and logical operators) in a step-by-step exploratory approach. Students responded positively to the worksheets, so they were adopted going forward. Extensive reading sets were eliminated and instead the instructor relayed the theoretical background using relatable examples, in one instance using data about a popular artist's ticket sales.

Some limitations do deserve comment. Most of the students (80\%) reported that they wished to continue the program. As this was a summer-only program, that was not possible. A more formal way to create an affirming space for coding could be a sustained effort in the future that carries the ideas from the summer program throughout the academic year. Also, due to the NPM structure, a limiting aspect of the program is recruiting a large number of NPMs that students can relate to, as there may be few at any given university. Recruiting NPMs from multiple sites to reach more students, like the crowdfunding solution proposed by Michael Johnson and colleagues ${ }^{2}$, may be a potential way to expand the program.

Models like VBRC could be developed and tested further as a potential solution to morph computer science programs, 
bioinformatics programs and biomedical programs into becoming more inclusive and affirming of BIPOC students, their interests and their lived experiences. This is certainly not the only program designed as an inclusive summer program; for example, programs such as the Big Data Summer Program, Meyerhoff, MARC and RISE also focus on diversity and inclusion. As VBRC was held online, it allows for easy scaling, which can be beneficial. With multiple, combined and sustained efforts like these, we can begin to tear down the structures of racism in education, with the goal of ultimately changing the face of science.

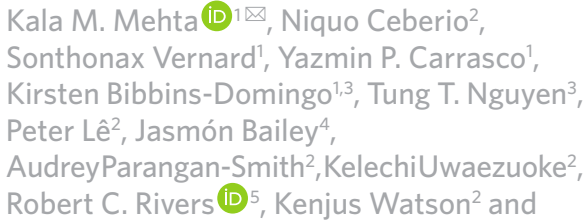

${ }^{1}$ Department of Epidemiology and Biostatistics, University of California San Francisco, San Francisco, CA, USA. ${ }^{2}$ Department of Biology, San Francisco State University, San Francisco, CA, USA. ${ }^{3}$ Department of Medicine, University of California San Francisco, San Francisco, CA, USA. ${ }^{4}$ Department of Sociology, University of Maryland, College Park, MD, USA. ${ }^{5}$ Office of Minority Health Research Coordination, National Institute of Diabetes and Digestive and Kidney Diseases, National Institutes of Health, Bethesda, MD, USA.

$凶_{e-m a i l: k a l a . m e h t a @ u c s f . e d u}$

Published online: 15 April 2021

https://doi.org/10.1038/s43588-021-00063-6

References

1. Bibbins-Domingo, K. Ann. Intern. Med. 173, 233-234 (2020).

2. Johnson, M. D. L., Baltrus, D. A. \& Gardy, J. Nat. Microbiol. 5 , 1311-1313 (2020)

Acknowledgements

We would like to thank SF BUILD staff members D. Espinosa, M. Guadalupe De Paz Fernandez, and SF
BUILD student assistants K. Leong and B. Garibay for their work on VBRC. We would like to acknowledge all members of the NIH BUILD Research Collaboratory team (faculty, students and staff) for their collaboration. We greatly appreciate the support of A. Gammie and E. Falcón-Morales the project officers of the NIH BUILD program. We also would like to acknowledge site-level collaboration at each of the NIH BUILD sites: San Francisco State University, California State Long Beach, California State Northridge, Morgan State University Baltimore, Portland State University, Mercy Detroit and University of Texas at El Paso. Lastly, we wish to thank the Big Data Summer Program (Pennings, Rohlfs). This work was funded by a grant from the NIH Common Fund through linked awards: GM118984, 11895 and 11896.

\section{Author contributions}

K.M.M., N.C., S.V., Y.P.C., K.B.D., J.B., A.P.S., K.U., K.W. and L.M.M. designed the work. K.M.M., N.C., P.L. and K.U. drafted the article. K.M.M., N.C., K.B.D., T.T.N., P.L., J.B., A.P.S., K.U., R.C.R., K.W. and L.M.M. revised the article. All authors approved the final article.

Competing interests

The authors declare no competing interests. 